\title{
Fault-Tolerant Quantum Dynamical Decoupling
}

\author{
K. Khodjasteh \\ Department of Physics, and Center for Quantum Information and Quantum Control, University of Toronto, 60 St. George St., Toronto, \\ ON, M5S 1A7, Canada \\ kaveh@physics.utoronto.ca \\ D.A. Lidar \\ Chemical Physics Theory Group, Chemistry Department, and Center for Quantum Information and Quantum Control, University of \\ Toronto, 80 St. George St., Toronto, ON, M5S 3H6, Canada \\ dlidar@chem.utoronto.ca
}

\begin{abstract}
We review our work concerning a method of decoherence control via concatenated dynamical decoupling (DD) pulses. These recursively nested DD pulse sequences exhibit a faulttolerance threshold similar to that of concatenated quantum error correcting codes. We briefly discuss how quantum logic gates can be incorporated into this framework.

(C)2005 Optical Society of America

OCIS codes: (030.1640) Coherence; (270.1670) Coherent optical effects; (320.5540) Pulse shaping
\end{abstract}

\section{Introduction}

Ideal quantum computers are fully controllable and isolated systems with a tensor product structure. Realistically neither controllability, isolation, nor even the tensor decomposition can be achieved easily in laboratory settings. Quantum operations tend to be faulty and furthermore the environment interacts with the system and produces undesirable decoherence, thus reducing the fidelity.

Dynamical decoupling (DD) is one of the (in-principle) universal methods originally introduced in order to overcome decoherence-errors [1], but not faulty controls. The method has been known and used in nuclear magnetic resonance (NMR) under different names and manifestations essentially ever since the Hahn spin echo experiment [2], for decoupling or recoupling of various interactions between nuclear spins. DD uses repetition of fast and strong pulses that effectively weaken undesired parts of the Hamiltonian, including couplings to an unknown environment, so as to reduce decoherence and increase fidelity. The theoretical considerations are often accompanied by the requirements that the pulses need to be, ideally, of zero width, and the period of the pulse-cycle has to be smaller than the system-bath evolution periods and decoherence time [3]. It has been further shown that in principle it is possible to use bounded-strength (yet strong) pulses [4]. It must be stressed that, with the exception of [4], schemes involving periodic pulse sequences have largely ignored the consequences of pulse imperfections.

In contrast, in quantum error correcting codes (QECCs), it is possible to address imperfections via concatenation. This is a technique in which the self-similar structure of recursively combined codes allows for an error-rate threshold below which arbitrary protection might be achieved [5]. In the non-Markovian noise regime the error correction threshold is much more stringent [6], while DD provides far less resource-hungry protection, demanding only fast pulses. This renders DD particularly appealing for solid-state semiconductor spin-based QIP implementations, where coupling to spin impurities constitutes an important and rate-limiting non-Markovian source of decoherence [7].

Completing the analogy between DD and QECCs, we review our work [8] that introduces a concatenation scheme for generating significantly more robust and effective DD pulse sequences than the previously known schemes based on serial DD (SDD) [1, 4]. We discuss concatenated DD (CDD) for bounded-spectrum baths (such as a spin-bath), using boundedstrength controls, while assuming a realistic control scenario. We also hint at ways of incorporating quantum logic gates, as required in a quantum computer subject to dynamical decoupling. 


\section{Dynamical decoupling and Concatenation}

Our main focus is the concept of an "effective Hamiltonian" associated with a propagator (unitary evolution operator) for a system. We assume that the system's Hamiltonian is the sum of intrinsic $\left(H_{S}^{\text {int }}\right)$ and time-dependent controllable $\left(H_{P}\right)$ Hamiltonians. We denote by $H_{e}$ the "else" Hamiltonian: the sum of all non-controllable terms including those describing the system-environment interactions in the larger system plus bath Hamiltonian. Ideal pulses refer to propagators corresponding to unitary evolutions of the system only. Non-ideal pulses includes two sources of errors in navigating the quantum system: (i) Random or systematic deviations $\left(W_{P}\right)$ from the intended $H_{P}$. (ii) The presence of $H_{e}$ during the pulse. While it is possible to formulate DD in terms of general Hamiltonian interactions, here we focus on a qubit system where any Hamiltonian can be written as

$$
H_{e}=X \otimes B_{X}+Y \otimes B_{Y}+Z \otimes B_{Z}
$$

where $X, Y$, and $Z$ are Pauli operators on the system and the $B_{i}$ are environment operators (tracefull terms are ignored: they have no dynamical effect). The simplest SDD scenario is the "parity kick", which we shall refer to as canonical DD: Consider the repetition of the pulse sequence $\mathrm{Xf}_{\tau} \mathrm{Xf}_{\tau}$, where $\mathrm{X}$ denotes the application of the pulse $X$, and $\mathrm{f}_{\tau}$ denotes a pulse-free evolution period of duration $\tau$. If the $X$ pulse acts ideally the action of the pulse produces the following propagator for the system and the environment: $I_{E}=X \exp \left(-i \tau H_{e}\right) X \exp \left(-i \tau H_{e}\right)=\exp \left(-i \tau X H_{e} X\right) \exp \left(-i \tau H_{e}\right)$, and ignoring $O\left(\tau^{2} H_{e}^{2}\right)$, we can write $I_{E}=\exp \left(-i 2 \tau H_{\text {eff }}\right)$, where $H_{\text {eff }}=\left(X H_{e} X+H_{e}\right) / 2$. Universal decoupling is achieved when $H_{\text {eff }}=0$. This is achieved for a qubit by including two smaller canonical DD cycles inside another one:

$$
\mathrm{Y} \underbrace{\mathrm{f}_{2 \tau}}_{\mathrm{Zf_{ \tau }} Z \mathrm{Zf}_{\tau}} \mathrm{Y} \underbrace{\mathrm{f}_{2 \tau}}_{\mathrm{Zf_{ \tau }} \mathrm{Zf_{ \tau }}}=\mathrm{Xf}_{\tau} \mathrm{Zf}_{\tau} \mathrm{Xf}_{\tau} \mathrm{Zf_{ \tau }}
$$

which transforms $H_{e}$ into an effective Hamiltonian that vanishes up to $O\left(\tau^{2} H_{e}^{2}\right)$.

Using Eq. (1), it is not hard to see that the $X$ canonical cycle has a geometric interpretation as the projection of the 3-dimensional vector-like quantity $\left(B_{X}, B_{Y}, B_{Z}\right)$ onto the $x$ axis. Similarly nesting the $Y$ and $Z$ cycles corresponds to successive projections of a vector onto the $y$ and $z$ axes, resulting in universal decoupling: $H_{\text {eff }}=0$. A simple application of the $\mathrm{BCH}$ formula shows that the $O\left(\tau^{2} H_{e}^{2}\right)$ terms also have a geometric interpretation as rotations following each projection: the effective Hamiltonian of a $P_{i}$ canonical cycle is given up to $O\left(\tau^{3} H_{e}^{3}\right)$ by $H_{\text {eff }}$,

$$
\frac{1}{2} H_{\mathrm{eff}, i}=: \mathcal{D}_{P_{i}}(\tau)\left[H_{e}\right]=\exp \left(-i \tau H_{i}^{\perp} / 2\right) H_{i}^{\|} \exp \left(i \tau H_{i}^{\perp} / 2\right)+O\left(\lambda^{3}\right),
$$

where $H_{i}^{\|}\left(H_{i}^{\perp}\right)$ is the component of $H_{e}$ that (anti-)commutes with $P_{i}$ (which is exhaustive for qubit systems), and $\lambda_{i}^{3}:=$ $\tau^{3}\left\|H_{i}^{\perp}\right\|^{2}\left\|H_{i}^{\|}\right\| \ll 1$. This extra rotation produces extra terms besides $H_{i}^{\|}$, hence imperfect $D D$. Despite the imperfection, this mapping cannot increase the magnitude of $H_{e}$, and typically decreases it [8].

One can now envisage a scheme in which multiple canonical cycles are nested inside each other and rapidly decrease the magnitude of $H_{e}$, and thus provide a systematic decoupling. Each universal DD cycle consists of a concatenation of canonical DDPS. We call this the "inner concatenation". Formally, the different canonical cycles in an inner concatenation exhaust all possible terms in $H_{e}$, should there be no rotations like Eq. (3). Once an inner concatenation cycle is found, these pulse cycles can now be concatenated within one another, while keeping the total experiment time $T \propto \tau$ fixed to obtain higher order pulse sequences ps (n). Here are the first two examples, simplified using Pauli-group identities:

$$
\begin{aligned}
& \operatorname{ps}(1)=\operatorname{XfZfXfZf}
\end{aligned}
$$

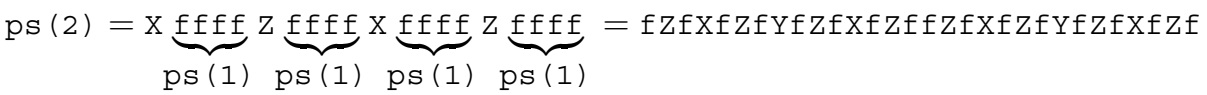

Clearly, given a fixed duration $\tau$, the pulse-free periods inbetween the pulses rapidly shorten, and given the actual pulse-width lower bound it is impossible to perform an arbitrarily high-order CDD. Furthermore, the number of pulses increases exponentially with the order of concatenation. While practically this is an issue, we can show that in the limit of zero-width pulses the success of CDD scales super-exponentially well, using an exponential number of pulses, thus providing an effective exponential advantage in terms of the number of pulses used. The "error"-phase associated with the effective Hamiltonian corresponding to deviation from ideal evolution (a simple measure of infidelity), satisfies [8]: $\left|\Phi_{\mathrm{CDD}}\right| \leq 4^{-\left[(2 n-1) 2^{n}+1\right]}\left\|\tau H_{0}\right\|^{2^{n}}$, where $n$ is the number of concatenation levels and $H_{0}=H_{e}$ is the "else" Hamiltonian before concatenation. A similar analysis for SDD, using the same number of pulses and minimum switching times yields 
$\left\|\Phi_{\mathrm{SDD}}\right\| \leq 4^{-n}\left\|\tau H_{0}\right\|^{2}$. Thus, while an exponential number of pulses is used, CDD converges super-exponentially, while SDD is only exponentially convergent. This difference is very similar to that between concatenated and serial QECC [5], and is indeed our motivation for CDD. While the above approximations are only suggestive in the case of finite-width pulses, our simulations show that there can be a saturation level for SDD after which squeezing in more pulses will not improve SDD at all, whereas CDD continues to improve [8].

\section{Non-ideal pulses, Fault-tolerance and Computation}

In practice every pulse has a non-zero width during which control jitters and $H_{e}$ produce deviations from the ideal action. Such deviations must be made small to allow for successful CDD. We give an upper bound on how these errors may be tolerated in a CDD pulse sequence, based on our geometric picture of the mapping of the Hamiltonians. The errors associated with imperfect pulses cause deviations in the effective Hamiltonian at each level of concatenation. They can thus be combined into the effective Hamiltonian after each level of concatenation (starting with the ones with shortest periods), and can thus can be removed by the next level of concatenation, if they are small enough. The condition is [8]:

$$
\delta\left(\gamma\left\|H_{e}\right\|+\left\langle\left|w_{P}\right|\right\rangle\left\|H_{P}\right\|\right) \ll \tau\left\|H_{e}\right\|
$$

where $\gamma \approx 1,\left\|W_{P}\right\|=\left|w_{P}\right|\left\|H_{P}\right\|, w_{P}$ is a dimensionless measure of the control jitter and $\delta$ is the pulse width. The left-hand side of this inequality gives the undesired phase accumulated during the pulse, which must be smaller than the phase due to $H^{\perp}$ (and hence $H_{e}$ ) accumulated during the free evolution. This must hold at every level of concatenation, in particular the deepest level where $\tau$ is smallest.

Should the above requirements be met, we would next like to consider incorporating quantum logic operations, allowing for fault-tolerant quantum computation. There seem to be several ways of doing this, and we mention one in this summary that uses an encoding of one qubit into a stabilizer code [9]. Stabilizer codes have the property that they detect any error that anticommutes with an element of the stabilizer. Such anticommutation is precisely the property required for a canonical DD cycle. It therefore turns out that for all stabilizer codes used for QECC, the stabilizer elements can be used as DD pulses to generate canonical DD cycles, providing DD of QECC codewords. Quantum operations can now be incorporated into the free evolution periods and can be made fault-tolerant, if the source of the errors is Hamiltonian coupling to a bounded environment. The stabilizer formalism and the encoding in this case are crucial, for otherwise DD practically removes all propagators from the system, including the unitary operations needed for quantum computation. The effect of DD in the encoded case is to bring the dynamics of the system as close as possible to a unitary operator on the encoded subspace, by removing all the error terms.

\section{References}

[1] L. Viola, E. Knill and S. Lloyd, "Dynamical Decoupling of Open Quantum Systems”, Phys. Rev. Lett. 82, 2417 (1999); P. Zanardi, “Symmetrizing Evolutions”, Phys. Lett. A 258, 77 (1999).

[2] E.L. Hahn, "Spin Echoes”, Phys. Rev. 80, 580 (1950).

[3] P. Facchi, D.A. Lidar, and S. Pascazio, "Unification of Dynamical Decoupling and the Quantum Zeno Effect", Phys. Rev. A 69, 032314 (2004).

[4] L. Viola and E. Knill, "Robust dynamical decoupling with bounded controls", Phys. Rev. Lett. 90, 037901 (2003).

[5] A.M. Steane, "Overhead and noise threshold of fault-tolerant quantum error correction", Phys. Rev. A 68, 042322 (2003); E. Knill, “Quantum Computing with Very Noisy Devices”, eprint quant-ph/0410199.

[6] B.M. Terhal, G. Burkard, "Fault-Tolerant Quantum Computation For Local Non-Markovian Noise", eprint quant$\mathrm{ph} / 0402104$.

[7] R. de Sousa, S. Das Sarma, "Theory of nuclear-induced spectral diffusion: Spin decoherence of phosphorus donors in Si and GaAs quantum dots", Phys. Rev. B 68, 115322 (2003).

[8] K. Khodjasteh, D.A. Lidar, “Concatenated Dynamical Decoupling”, eprint quant-ph/0408128.

[9] D. Gottesman, "Class of quantum error-correcting codes saturating the quantum Hamming bound", Phys. Rev. A 54, 1862 (1996). 